\title{
$F$-STABILITY OF $f$-MINIMAL HYPERSURFACE
}

\author{
WEIMIN SHENG AND HAOBIN YU
}

(Communicated by Lei $\mathrm{Ni}$ )

\begin{abstract}
In this paper we study the classification of the $f$-minimal hypersurface immersed in the manifold $M^{n} \times R$, where $\left(M^{n}, g\right)$ is an Einstein manifold with positive Ricci curvature. By using the $F$ functional and $F$ stability which were introduced by Huisken and Colding-Minicozzi respectively, we prove that among all complete $f$-minimal hypersurfaces with polynomial volume growth, only $M^{n} \times\{0\}$ is $F$-stable.
\end{abstract}

\section{INTRODUCTION}

A hypersurface $\Sigma$ immersed into a Riemannian manifold $(\bar{M}, \bar{g})$ is called an $f$ minimal hypersurface if it satisfies that, for any point $p \in \Sigma$,

$$
H=\langle\bar{\nabla} f, \nu\rangle,
$$

where $H$ is the mean curvature, $f$ is a smooth function defined on $\bar{M}, \bar{\nabla} f$ is the gradient of $f$ on $\bar{M}$, and $\nu$ is the outer unit normal. In fact, if a hypersurface $\Sigma$ satisfies (1.1), then $\Sigma$ is a minimal hypersurface of $\bar{M}$ with respect to the conformal metric $e^{-\frac{2 f}{n}} \bar{g}$.

Let $\Sigma$ be a hypersurface immersed into $R^{n+1}$. Given $x_{0} \in R^{n+1}$ and $t_{0} \geq 0$, Huisken [7] defined the functional $F_{x_{0}, t_{0}}$ for a mean curvature flow $\Sigma_{t}, t<t_{0}$, by

$$
F_{x_{0}, t_{0}}=\left(4 \pi\left(t_{0}-t\right)\right)^{-\frac{n}{2}} \int_{\Sigma_{t}} e^{-\frac{\left|x-x_{0}\right|^{2}}{4\left(t_{0}-t\right)}} d \mu
$$

and proved the functional is monotonically decreasing under the mean curvature flow. In [12, John Lott proved that Huisken's monotonicity holds when the ambient manifold is a gradient Ricci soliton. He also showed that the mean curvature soliton immersed into gradient Ricci solitons is just the $f$-minimal hypersurface, where $f$ is the potential function. Recently, $f$-minimal hypersurfaces have been studied extensively; see 2, 4, 10,11 for instance.

A hypersurface immersed into $R^{n+1}$ is called a self-shrinker if it satisfies

$$
H=\langle x, \nu\rangle \text {. }
$$

Self-shrinker is one of the most important $f$-minimal hypersurfaces by letting $\bar{M}=$ $R^{n+1}$ and $f=\frac{|x|^{2}}{2}$ in (1.1), and plays a crucial role in the study of the singularity of the mean curvature flow. Using the functional (1.2), Colding and Minicozzi [6] classified the self-shrinkers with nonnegative mean curvature and polynomial

Received by the editors September 30, 2013.

2010 Mathematics Subject Classification. Primary 53C21; Secondary 53C23.

Key words and phrases. F-stability, $f$-minimal hypersurface, gradient Ricci soliton.

The authors were supported by NSFC, grant no. 11131007, and Zhejiang provincial natural science foundation of China, grant no. LY14A010019. 
volume growth. They showed that every self-shrinker is unstable in the usual sense, so they introduced the concept of $F$-stability in a natural way and classified the $F$-stable self-shrinkers with polynomial volume growth. In [9], Hussey classified the self-shrinkers with low index. For the harmonic heat flow, Y.-B. Zhang 14] considered the $F$-stability of self-similar solutions to the harmonic map flow.

In this paper, we consider the case that $\bar{M}=M^{n} \times R$, where $\left(M^{n}, g\right)$ is an Einstein manifold with positive Ricci curvature $\kappa$, and $f=\frac{\kappa z^{2}}{2}$. By Myers' Theorem [13] we know $\left(M^{n}, g\right)$ is compact. We denote a point $p \in \bar{M}$ by $\left(p^{\prime}, z\right)$, where $p^{\prime} \in M^{n}$ and $z \in R$. Let $\bar{g}=g+d z^{2}$ be the product metric on $M^{n} \times R$. Thus, $(\bar{M}, \bar{g}, f)$ is a shrinking gradient Ricci soliton, and

$$
\overline{R i c}+\bar{\nabla}^{2} f=\kappa \bar{g} .
$$

Now a hypersurface immersed in $M^{n} \times R$ is an $f$-minimal hypersurface if it satisfies

$$
H=\kappa z\langle\partial z, \nu\rangle \text {. }
$$

We have

Theorem 1.1. Let $\Sigma \subset M^{n} \times R$ be a complete hypersurface with $H=\kappa z\langle\partial z, \nu\rangle$. Assume $\langle\partial z, \nu\rangle \geq 0$.

(1) If $\Sigma$ is compact, then $\langle\partial z, \nu\rangle \equiv 1$, and $\Sigma=M^{n} \times\{0\}$.

(2) If $\Sigma$ is complete noncompact and has polynomial volume growth, then $\langle\partial z, \nu\rangle \equiv 0$, and $\Sigma=\Sigma^{\prime} \times R$. In this case, $\Sigma^{\prime}$ is a minimal hypersurface in $M^{n}$.

Given $a \in R$, we define the functional $F_{a}$ by

$$
F_{a}(\Sigma)=\int_{\Sigma} e^{-\frac{\kappa\left(z^{2}-2 a z+a^{2}\right)}{2}} d \mu
$$

We will show that the $f$-minimal hypersurface in our case is the critical point of the $F_{a}$-functional defined by (1.3). Now we can introduce the concept of the stability of the $F_{a}$-functional for the $f$-minimal hypersurfaces. A critical point $\Sigma$ for the $F_{a}$-functional is $F$-stable if for any compactly supported variation $\Sigma_{s}$ with $\Sigma_{0}=\Sigma$, there exists variation $a_{s}$ of $a$ that makes $F^{\prime \prime}=\left(F_{a_{s}}\left(\Sigma_{s}\right)\right)^{\prime \prime} \geq 0$ at $s=0$. We have that

Theorem 1.2. $M^{n} \times\{0\}$ is the only compact F-stable hypersurface in $M^{n} \times R$.

Theorem 1.3. If $\Sigma \subset M^{n} \times R$ is a complete noncompact hypersurface with polynomial volume growth and satisfies $H=\kappa z\langle\partial z, \nu\rangle$, then $\Sigma$ is $F$-unstable.

In 4, by using the $L_{f}$-index, Cheng and Zhou studied the $f$-minimal hypersurfaces in a cylinder shrinker soliton $\left(S^{n}(\sqrt{2(n-1)}) \times R, \bar{g}, f\right)$ and showed that such hypersurface with $L_{f}$-index one must be either $S^{n}(\sqrt{2(n-1)}) \times\{0\}$ or $S^{n-1}(\sqrt{2(n-1)}) \times R$. By our Theorem 1.2. $S^{n}(\sqrt{2(n-1)}) \times\{0\}$ is the only compact $F$-stable hypersurface in $S^{n}(\sqrt{2(n-1)}) \times R$.

The rest of this paper is organized as follows. In Section 2, we compute the first variation formula of the $F_{a}$-functional. In Section 3 , we compute the second variation formula of the $F_{a}$-functional at the critical point and we present the proof of Theorem 1.1. In Section 4, we consider the $F$-stable $f$-minimal hypersurface and give the proofs of Theorem 1.2 and Theorem 1.3 . 


\section{First variation and $f$-minimal hypersurface}

Lemma 2.1. Let $\Sigma_{s} \subset \bar{M}$ be a variation of the hypersurface $\Sigma=\Sigma_{0}$ with variation vector field $\Sigma_{0}^{\prime}=\varphi \nu$, and let $a_{s} \in R$ be the variation of a with $a^{\prime}=h$. Then

$$
\left.\frac{\partial}{\partial s}\right|_{s=0} F_{a_{s}}\left(\Sigma_{s}\right)=\int_{\Sigma}[\varphi(H-\kappa z\langle\nu, \partial z\rangle+a \kappa\langle\partial z, \nu\rangle)+\kappa h(z-a)] e^{-\frac{\kappa}{2}(z-a)^{2}} d \mu .
$$

Proof. Using $\left.\frac{\partial X_{s}}{\partial s}\right|_{s=0}=\varphi \nu$ and $\left.\frac{\partial a_{s}}{\partial s}\right|_{s=0}=h$, we get

$$
(d \mu)^{\prime}=\varphi H d \mu \text {. }
$$

The derivative of $e^{-\frac{\kappa}{2}\left(z-a_{s}\right)^{2}}$ at $s=0$ is

$$
-e^{-\frac{\kappa}{2}(z-a)^{2}} \kappa(z-a)(\varphi\langle\partial z, \nu\rangle-h) .
$$

Combining (2.2) and (2.3) gives the desired result.

Let $\Sigma \subset M^{n} \times R$ be a smooth immersed hypersurface, and let $\Delta$ and $\nabla$ be the Laplacian and gradient on $\Sigma$, respectively. We define the linear elliptic operator as follows:

$$
\ell v=\Delta v-\kappa z\langle\partial z, \nabla v\rangle
$$

where $v$ is any smooth function on $\Sigma$. By Stokes' theorem, we have the following.

Lemma 2.2. If $\Sigma \subset M^{n} \times R$ is a hypersurface, $u \in C_{c}^{1}(\Sigma)$, and $v \in C^{2}(\Sigma)$, then

$$
\int_{\Sigma} u(\ell v) e^{-\frac{\kappa z^{2}}{2}}=-\int_{\Sigma}\langle\nabla u, \nabla v\rangle e^{-\frac{\kappa z^{2}}{2}}
$$

By a similar argument as in Corollary 3.10 in [6], we can obtain

Corollary 2.1. Suppose $\Sigma \subset M^{n} \times R$ is a complete hypersurface, and $u$ and $v$ are $C^{2}$ functions satisfying

$$
\int_{\Sigma}(|u \nabla v|+|\nabla u||\nabla v|+|u \ell v|) e^{-\frac{\kappa z^{2}}{2}}<\infty
$$

Then

$$
\int_{\Sigma} u(\ell v) e^{-\frac{\kappa z^{2}}{2}}=-\int_{\Sigma}\langle\nabla u, \nabla v\rangle e^{-\frac{\kappa z^{2}}{2}}
$$

Next we show the behaviour of $\ell$ on the coordinate function.

Lemma 2.3. If $\Sigma \subset M^{n} \times R$ is a hypersurface with $H=\kappa z\langle\partial z, \nu\rangle$, then

$$
\begin{gathered}
\ell z=-\kappa z, \\
\ell z^{2}=2-2 \kappa z^{2}-2\langle\partial z, \nu\rangle^{2} .
\end{gathered}
$$

Proof. We denote by $\bar{\Delta}$ and $\bar{\nabla}$ the Laplacian and gradient on $\bar{M}$, respectively. Then

$$
\begin{gathered}
\Delta z=\bar{\Delta} z-\left(\bar{\nabla}^{2} z\right)(\nu, \nu)-H\langle\bar{\nabla} z, \nu\rangle, \\
\bar{\Delta} z=0, \quad \bar{\nabla}^{2} z(\nu, \nu)=0 .
\end{gathered}
$$

Using $H=\kappa z\langle\partial z, \nu\rangle$, we obtain

$$
\begin{aligned}
\ell z & =\Delta z-\kappa z\langle\partial z, \nabla z\rangle \\
& =-\kappa z\langle\partial z, \nu\rangle^{2}-\kappa z\left\langle(\partial z)^{T},(\partial z)^{T}\right\rangle \\
& =-\kappa z .
\end{aligned}
$$


By the same argument,

$$
\begin{gathered}
\Delta z^{2}=\bar{\Delta} z^{2}-\left(\bar{\nabla}^{2} z^{2}\right)(\nu, \nu)-H\left\langle\bar{\nabla} z^{2}, \nu\right\rangle, \\
\bar{\Delta} z^{2}=2, \quad \bar{\nabla}^{2} z^{2}(\nu, \nu)=2\langle\partial z, \nu\rangle^{2},
\end{gathered}
$$

and we get

$$
\begin{aligned}
\ell z^{2} & =\Delta z^{2}-\kappa z\left\langle\nabla z^{2}, \partial z\right\rangle \\
& =2-2\langle\partial z, \nu\rangle^{2}-2 \kappa z^{2}\langle\partial z, \nu\rangle^{2}-2 \kappa z^{2}\left\langle(\partial z)^{T},(\partial z)^{T}\right\rangle \\
& =2-2 \kappa z^{2}-2\langle\partial z, \nu\rangle^{2} .
\end{aligned}
$$

Corollary 2.2. If $\Sigma \subset M^{n} \times R$ is a hypersurface with $H=\kappa z\langle\partial z, \nu\rangle$ and polynomial volume growth, then

$$
\begin{gathered}
\int_{\Sigma} z e^{-\frac{\kappa z^{2}}{2}}=0 \\
\int_{\Sigma} \kappa z^{2} e^{-\frac{\kappa z^{2}}{2}}=\int_{\Sigma}\left(1-\langle\partial z, \nu\rangle^{2}\right) e^{-\frac{\kappa z^{2}}{2}} .
\end{gathered}
$$

Proof. Using $|\nabla z| \leq 1,|\ell z|=\kappa|z|$ and the polynomial volume growth of $\Sigma$, we have

$$
\int_{\Sigma} e^{-\frac{\kappa z^{2}}{2}}<\infty \text { and } \int_{\Sigma} \kappa|z| e^{-\frac{\kappa z^{2}}{2}}<\infty
$$

Letting $u=1$ and $v=z$ in Corollary 2.1 gives (2.4).

Similarly, we can get $\left|\nabla z^{2}\right| \leq 2|z|,\left|\ell z^{2}\right| \leq 2+2 \kappa z^{2}$, and

$$
\int_{\Sigma}\left(2 \kappa z^{2}+2\right) e^{-\frac{\kappa z^{2}}{2}}<\infty \text {. }
$$

Now setting $u=1$ and $v=z^{2}$ in Corollary 2.1 yields (2.5).

Remark 2.1. If the Ricci curvature of $M^{n}$ is negative, i.e., $\kappa<0$, and assuming $\Sigma$ is a compact hypersurface, then $\langle\partial z, \nu\rangle \equiv 0$ and $z(x) \equiv 0$ for any point $x \in \Sigma$, so $\Sigma=M^{n} \times\{0\}$. In particular, if $M^{n}$ is a complete noncompact Einstein manifold with negative Ricci curvature, then there exists no compact $f$-minimal hypersurface in $M^{n} \times R$.

By Lemma 2.1 and Corollary 2.2, we can get the following.

Proposition 2.1. $\Sigma$ is a critical point for $F_{a}(\Sigma)$ if and only if $H=\kappa z\langle\partial z, \nu\rangle-$ $a \kappa\langle\partial z, \nu\rangle$.

Proof. For any point $x=\left(x^{\prime}, z\right) \in \bar{M}$, let $\tilde{x}=\left(x^{\prime}, z-a\right)$ and $\tilde{\Sigma}=\left\{\tilde{x}=\left(x^{\prime}, z-a\right) \mid x \in\right.$ $\bar{M}\}$. If $\Sigma$ satisfies

$$
H=\kappa z\langle\partial z, \nu\rangle-a \kappa\langle\partial z, \nu\rangle,
$$

then

$$
H(\tilde{x})=\kappa z(\tilde{x})\langle\partial z, \nu\rangle
$$

for any point $\tilde{x} \in \tilde{\Sigma}$, and

$$
\int_{\Sigma} \kappa h(z-a) e^{-\frac{\kappa(z-a)^{2}}{2}}=\int_{\tilde{\Sigma}} z e^{-\frac{\kappa z^{2}}{2}}=0,
$$

where the second equality follows from Corollary 2.2. 
3. SECOND VARIATion OF $F_{a}(\Sigma)$

Lemma 3.1. If $\Sigma_{s}$ is a normal variation of $\Sigma$, and $a_{s}$ is a variation of $a_{0}$ with

$$
\begin{aligned}
& \left.\partial_{s}\right|_{s=0} \Sigma_{s}=\varphi \nu,\left.\partial_{s}\right|_{s=0} a_{s}=h, \\
& \left.\partial_{s}\right|_{s=0} \varphi=\varphi^{\prime},\left.\partial_{s s}\right|_{s=0} a_{s}=h^{\prime},
\end{aligned}
$$

then, setting $F^{\prime \prime}=\left.\partial_{s s}\right|_{s=0}\left(F_{a_{s}}\left(\Sigma_{s}\right)\right)$, we have

$$
\begin{aligned}
F^{\prime \prime}= & \int_{\Sigma}\left\{\left(-\varphi L_{a_{0}} \varphi+2 \kappa h \varphi\langle\partial z, \nu\rangle-\kappa h^{2}\right)\right. \\
& +\left(\varphi\left(H-\kappa z\langle\partial z, \nu\rangle+a_{0} \kappa\langle\partial z, \nu\rangle\right)+\kappa h\left(z-a_{0}\right)\right)^{2} \\
& \left.+\varphi^{\prime}\left(H-\kappa z\langle\partial z, \nu\rangle+a_{0} \kappa\langle\partial z, \nu\rangle\right)+\kappa h^{\prime}\left(z-a_{0}\right)\right\} e^{-\frac{\kappa}{2}\left(z-a_{0}\right)^{2}} d \mu .
\end{aligned}
$$

Here $L_{a_{0}}=\Delta-\kappa\left(z-a_{0}\right)\langle\nabla, \partial z\rangle+|A|^{2}+\kappa$.

Proof. We use primes to denote the derivatives with respect to $s$ at $s=0$. For any smooth function $\psi$ defined on $\Sigma$, we use [.] to denote the weighted integral, i.e.,

$$
[\psi]=\int_{\Sigma} \psi e^{-\frac{\kappa z^{2}}{2}} d \mu
$$

Then

$$
\begin{aligned}
F^{\prime \prime}= & {\left[\varphi\left(H-\kappa z\langle\nu, \partial z\rangle+a_{s} \kappa\langle\partial z, \nu\rangle\right)^{\prime}+\kappa\left(h z-a_{s} h\right)^{\prime}\right.} \\
& +\left(\varphi\left(H-\kappa z\langle\nu, \partial z\rangle+a_{0} \kappa\langle\partial z, \nu\rangle\right)+\kappa\left(h z-a_{0} h\right)\right)^{2} \\
& \left.+\varphi^{\prime}\left(H-\kappa z\langle\nu, \partial z\rangle+a_{0} \kappa\langle\partial z, \nu\rangle\right)\right] .
\end{aligned}
$$

By standard calculations (see [8]), we can get

$$
\begin{aligned}
& H^{\prime}=-\Delta \varphi-|A|^{2} \varphi-\overline{\operatorname{Ric}}(\nu, \nu) \varphi \\
& \nu^{\prime}=-\nabla \varphi .
\end{aligned}
$$

Note that

$$
\overline{R i c}+\bar{\nabla}^{2}\left(\frac{\kappa z^{2}}{2}\right)=\kappa \bar{g},
$$

so

$$
\overline{\operatorname{Ric}}(\nu, \nu)+\bar{\nabla}^{2} \frac{\kappa z^{2}}{2}(\nu, \nu)=\overline{\operatorname{Ric}}(\nu, \nu)+\kappa\langle\partial z, \nu\rangle^{2}=\kappa .
$$

Now we have

$$
\begin{aligned}
& \left(H-\kappa z\langle\nu, \partial z\rangle+a_{s} \kappa\langle\partial z, \nu\rangle\right)^{\prime} \\
= & -\Delta \varphi-|A|^{2} \varphi-\overline{\operatorname{Ric}}(\nu, \nu) \varphi-\kappa \varphi\langle\partial z, \nu\rangle^{2}+\kappa z\langle\partial z, \nabla \varphi\rangle \\
& -a_{0} \kappa\langle\partial z, \nabla \varphi\rangle+h \kappa\langle\partial z, \nu\rangle \\
= & -L_{a_{0}} \varphi+h \kappa\langle\partial z, \nu\rangle .
\end{aligned}
$$

The second term is given by

$$
\kappa\left(h z-a_{s} h\right)^{\prime}=\kappa\left(h^{\prime} z+h \varphi\langle\partial z, \nu\rangle-h^{2}-a_{0} h^{\prime}\right) .
$$

Combining (3.2), (3.5), and (3.6) gives the lemma. 
Theorem 3.1. Suppose $\Sigma$ is a complete hypersurface, has polynomial volume growth, and satisfies $H=\kappa z\langle\partial z, \nu\rangle$. If $\Sigma_{s}$ is the normal variation of $\Sigma, a_{s} \in R$ is the variation with $a_{0}=0$, and

$$
\left.\partial_{s}\right|_{s=0} \Sigma_{s}=\varphi \nu,\left.\partial_{s}\right|_{s=0} a_{s}=h
$$

then

$$
F^{\prime \prime}=\int_{\Sigma}\left(-\varphi L \varphi+2 \kappa h \varphi\langle\nu, \partial z\rangle-\kappa h^{2}\langle\partial z, \nu\rangle^{2}\right) e^{-\frac{\kappa z^{2}}{2}} d \mu .
$$

Here $L=\Delta-\kappa z\langle\partial z, \nabla \cdot\rangle+|A|^{2}+\kappa$.

Proof. Since $H=\kappa z\langle\partial z, \nu\rangle$ and $a_{0}=0$, by Lemma 3.1,

$$
F^{\prime \prime}=\int_{\Sigma}\left(-\varphi L \varphi+2 \kappa h \varphi\langle\partial z, \nu\rangle-\kappa h^{2}+\kappa^{2} h^{2} z^{2}\right) e^{-\frac{\kappa z^{2}}{2}}
$$

where we have used (2.4). By (2.5), it follows that

$$
F^{\prime \prime}=\int_{\Sigma}\left(-\varphi L \varphi+2 \kappa h \varphi\langle\nu, \partial z\rangle-\kappa h^{2}+\kappa h^{2}\left(1-\langle\partial z, \nu\rangle^{2}\right) e^{-\frac{\kappa z^{2}}{2}} d \mu,\right.
$$

which completes the proof.

A hypersurface $\Sigma$ with $H=\kappa z\langle\partial z, \nu\rangle$ is a minimal hypersurface in $\left(\bar{M}, e^{-\frac{\kappa z^{2}}{n}} \bar{g}\right)$, and $\Sigma$ is called stable [5] in the usual sense if, for any function $\varphi$ with compact support,

$$
\int_{\Sigma}-\varphi L \varphi e^{-\frac{\kappa z^{2}}{2}} d \mu \geq 0
$$

In [1], Cheng-Mejia-Zhou proved that if $(\bar{M}, \bar{g}, f)$ is a complete manifold with $\overline{\text { Ric }_{f}} \geq c$, where $c$ is a positive constant, then there is no two-sided stable $f$-minimal hypersurface with finite weighted volume in $(\bar{M}, \bar{g}, f)$. In our case,

$$
\overline{\operatorname{Ric}}_{f}=\overline{\operatorname{Ric}}+\bar{\nabla}^{2} f=\kappa \bar{g} .
$$

Since $\kappa$ is a positive constant, there exists no stable complete $f$-minimal hypersurface with finite weighted volume. Now we introduce the following.

Definition 3.1. A hypersurface $\Sigma$ with $H=\kappa z\langle\partial z, \nu\rangle$ is called $F$-stable if, for any compactly supported function $\varphi$, there exists some constant $h$, such that $F^{\prime \prime} \geq 0$.

By definition, a stable $f$-minimal hypersurface must be $F$-stable. By Corollary 4 from [3], we have the following.

Lemma 3.2. $L\langle\partial z, \nu\rangle=\kappa\langle\partial z, \nu\rangle$.

We also need the following.

Lemma 3.3. Suppose $\psi$ is a $C^{2}$ function satisfying $L \psi=-\mu \psi$, for some $\mu \in R$. If $\psi>0$, then for any function $\varphi$ in the weighted $W^{1,2}$ space, we have

$$
\left[\varphi^{2}|A|^{2}\right] \leq\left[|\nabla \varphi|^{2}-\varphi^{2}(\mu+\kappa)\right] .
$$

Proof. Since $\psi>0$ and $\ln \psi$ is well defined,

$$
\ell \ln \psi=-|\nabla \ln \psi|^{2}+\frac{\Delta \psi-\kappa z\langle\partial z, \nabla \psi\rangle}{\psi}=-\mu-|A|^{2}-\kappa-|\nabla \ln \psi|^{2} .
$$

Suppose $\eta$ is a function with compact support. By Lemma 2.2,

$$
\left[\left\langle\nabla \eta^{2}, \nabla \ln \psi\right\rangle\right]=-\left[\eta^{2} \ell \ln \psi\right]=\left[\eta^{2}\left(\mu+|A|^{2}+\kappa+|\nabla \ln \psi|^{2}\right)\right] .
$$


Using

$$
\left\langle\nabla \eta^{2}, \nabla \ln \psi\right\rangle \leq|\nabla \eta|^{2}+\eta^{2}|\nabla \ln \psi|^{2}
$$

we get

$$
\left[\eta^{2}\left(\mu+|A|^{2}+\kappa+|\nabla \ln \psi|^{2}\right)\right] \leq\left[|\nabla \eta|^{2}+\eta^{2}|\nabla \ln \psi|^{2}\right],
$$

which yields

$$
\left[\eta^{2}|A|^{2}\right] \leq\left[|\nabla \eta|^{2}-\eta^{2}(\mu+\kappa)\right] .
$$

Let $\eta_{j}$ be 1 in $M^{n} \times(-j, j)$, and cut off linearly to zero in $M^{n} \times(-2 j, 2 j) \backslash M^{n} \times$ $(-j, j)$. Since $\varphi$ is in the weighted $W^{1,2}$ space, applying (3.8) with $\eta=\eta_{j} \varphi$, letting $j \rightarrow \infty$, and using the monotone convergence theorem gives (3.7).

Using Lemma 3.3. we may prove Theorem 1.1.

Proof of Theorem 1.1. (1) Consider the coordinate function $z$ on $\Sigma$. Since $\Sigma$ is compact, there exists a point $p \in \Sigma$ such that $z(p)=\max _{\Sigma} z$. So

$$
|\nabla z|^{2}(p)=1-\langle\partial z, \nu\rangle^{2}(p)=0 .
$$

Assume $\langle\partial z, \nu\rangle(p)=1$; then the Harnack inequality gives

$$
\langle\partial z, \nu\rangle(q)>0, \forall q \in \Sigma .
$$

By Lemma 3.2 ,

$$
\begin{aligned}
\ell\langle\partial z, \nu\rangle & =L\langle\partial z, \nu\rangle-\left(|A|^{2}+\kappa\right)\langle\partial z, \nu\rangle \\
& =-|A|^{2}\langle\partial z, \nu\rangle .
\end{aligned}
$$

Setting $u \equiv 1$ and $v=\langle\partial z, \nu\rangle$ in Lemma 2.2 gives

$$
\int_{\Sigma}|A|^{2}\langle\partial z, \nu\rangle e^{-\frac{\kappa z^{2}}{2}}=0
$$

Thus $|A|^{2} \equiv 0$, and $H=\kappa z\langle\partial z, \nu\rangle \equiv 0$. Since $\langle\partial z, \nu\rangle>0$, then $z \equiv 0 . \quad \Sigma$ is compact, so $\Sigma=M^{n} \times\{0\}$ and $\langle\partial z, \nu\rangle \equiv 1$.

(2) Suppose not. Then there exists some point $p \in \Sigma$ such that $\langle\partial z, \nu\rangle(p)>0$. Applying the Harnack inequality, we get $\langle\partial z, \nu\rangle>0$ in $\Sigma$. Obviously $\varphi \equiv 1$ is in the weighted $W^{1,2}$ space, so by Lemma 3.3, we know

$$
\int_{\Sigma}|A|^{2} e^{-\frac{\kappa z^{2}}{2}}<\infty .
$$

Setting $u=1$ and $v=\langle\partial z, \nu\rangle$ in Corollary [2.1, we obtain

$$
\int_{\Sigma}|A|^{2}\langle\partial z, \nu\rangle e^{-\frac{\kappa z^{2}}{2}}=0 .
$$

By the same argument as before, we find $\Sigma=M^{n} \times\{0\}$, which contradicts our assumption that $\Sigma$ is noncompact. Now $\langle\partial z, \nu\rangle \equiv 0$ and let

$$
\Sigma^{\prime}=\Sigma \cap\left(M^{n} \times\{0\}\right) ;
$$

then $\Sigma=\Sigma^{\prime} \times R$. Since

$$
H=\kappa z\langle\partial z, \nu\rangle \equiv 0,
$$

$\Sigma^{\prime}$ is a minimal hypersurface in $M^{n}$. 


\section{F-Stable $f$-Minimal hypersurface in $M^{n} \times R$}

In this section, we give the proofs of Theorem 1.2 and Theorem 1.3 ,

Proof of Theorem 1.2. Firstly, we show that $\Sigma=M^{n} \times\{0\}$ is F-stable. Now $H=0,|A|^{2}=0$, and $\nu=\partial z$. Then $L=\Delta+\kappa$ and

$$
F^{\prime \prime}=\int_{\Sigma}-\varphi(\Delta \varphi+\kappa \varphi)+2 \kappa h \varphi-\kappa h^{2} .
$$

We use $\lambda_{1}$ to denote the first nonzero eigenvalue of the Laplacian on $M$, then by Lichnerowicz's theorem, $\lambda_{1} \geq \frac{n \kappa}{n-1}$. The eigenfunctions corresponding to $\lambda_{0}=0$ are constant functions; let $E$ be the space of functions which are orthogonal to the constants, i.e.,

$$
E=\left\{\psi \in W^{1,2}(\Sigma): \int_{M^{n}} \psi=0\right\} .
$$

For any smooth function $\varphi$, choose $b \in R$ such that

$$
\varphi_{0} \equiv \varphi-b \in E .
$$

Now we get that

$$
F^{\prime \prime}=\int_{M^{n}}\left[-\left(\varphi_{0}+b\right) \Delta \varphi_{0}-\kappa\left(\varphi_{0}+b\right)^{2}+2 \kappa h\left(\varphi_{0}+b\right)-\kappa h^{2}\right] .
$$

By the orthogonality of the different eigenspaces, it follows that

$$
F^{\prime \prime} \geq \int_{M^{n}} \varphi_{0}^{2}\left(\lambda_{1}-\kappa\right)+\int_{M^{n}}-\kappa\left(b^{2}-2 b h+h^{2}\right) .
$$

Since $\lambda_{1}=\frac{n \kappa}{n-1}>\kappa$, then

$$
F^{\prime \prime} \geq \int_{M^{n}}-\kappa(b-h)^{2} .
$$

So $F^{\prime \prime}$ can be made nonnegative by choosing $h=b$.

We will show that if the compact hypersurface $\Sigma$ satisfies $H=\kappa z\langle\partial z, \nu\rangle$ and $\Sigma \neq M^{n} \times\{0\}$, then $\Sigma$ is $F$-unstable, i.e., there exists some function $\varphi$, such that for any constant $h$, we have $F^{\prime \prime}<0$.

Since $\Sigma \neq M^{n} \times\{0\}$, we know that $\mu_{1}$, the first eigenvalue of $L$, satisfies

$$
\mu_{1}<-\kappa \text {. }
$$

Let $\varphi$ be the eigenfunction corresponding to $\mu_{1}$ with $\int_{\Sigma} \varphi^{2} e^{-\frac{\kappa z^{2}}{2}}=1$. Since

$$
L\langle\partial z, \nu\rangle=\kappa\langle\partial z, \nu\rangle
$$

$\varphi$ and $\langle\partial z, \nu\rangle$ are orthogonal in the weighted $L^{2}$ space. For any constant $h$, it follows that

$$
F^{\prime \prime}=\int_{\Sigma}\left(\mu_{1} \varphi^{2}-\kappa h^{2}\langle\partial z, \nu\rangle\right) e^{-\frac{\kappa z^{2}}{2}} \leq \int_{\Sigma} \mu_{1} \varphi^{2} e^{-\frac{\kappa z^{2}}{2}}<0 .
$$

Now we consider the complete noncompact $f$-minimal hypersurface. Suppose $\Sigma \subset M^{n} \times R$ is a hypersurface with polynomial volume growth and satisfies $H=$ $\kappa z\langle\partial z, \nu\rangle$. 
Proof of Theorem 1.3. We will consider two separate cases.

Case 1: $\langle\partial z, \nu\rangle$ doesn't change sign, so we can assume $\langle\partial z, \nu\rangle \geq 0$. By Theorem 1.1. $\langle\partial z, \nu\rangle \equiv 0, \Sigma=\Sigma^{\prime} \times R$, and

$$
L=\Delta-\kappa z\langle\partial z, \nabla \cdot\rangle+|A|^{2}+\kappa .
$$

We use $\Delta^{\prime}$ to denote the Laplacian on $\Sigma^{\prime}$. Then

$$
L=\frac{\partial^{2}}{\partial z^{2}}+\Delta^{\prime}-\kappa z \frac{\partial}{\partial z}+|A|^{2}+\kappa .
$$

Use $\varphi(x)=\varphi\left(x^{\prime}, z\right)$ as a variation, where

$$
\varphi\left(x^{\prime}, z\right)= \begin{cases}1, & |z| \leq R \\ 2-\frac{|z|}{R}, & R \leq|z| \leq 2 R \\ 0, & |z| \geq 2 R\end{cases}
$$

where $R$ is a constant large enough to be determined. Then

$$
\begin{aligned}
F^{\prime \prime} & =\int_{\Sigma}-\varphi L \varphi e^{-\frac{\kappa z^{2}}{2}} \\
& =\int_{\Sigma}-\varphi\left(\frac{\partial^{2} \varphi}{\partial z^{2}}+\Delta^{\prime} \varphi-\kappa z \frac{\partial \varphi}{\partial z}+|A|^{2} \varphi+\kappa \varphi\right) e^{-\frac{\kappa z^{2}}{2}} \\
& =\int_{\Sigma}-\left(|A|^{2} \varphi^{2}+\kappa \varphi^{2}\right) e^{-\frac{\kappa z^{2}}{2}}+\int_{\Sigma \cap\{R \leq|z| \leq 2 R\}} \kappa z \varphi \frac{\partial \varphi}{\partial z} e^{-\frac{\kappa z^{2}}{2}} \\
& \leq \int_{\Sigma}-\left(|A|^{2} \varphi^{2}+\kappa \varphi^{2}\right) e^{-\frac{\kappa z^{2}}{2}}+\int_{\Sigma \cap\{R \leq|z| \leq 2 R\}} \kappa\left(\frac{2|z|}{R}+\frac{z^{2}}{R^{2}}\right) e^{-\frac{\kappa z^{2}}{2}}
\end{aligned}
$$

where we have used

$$
\frac{\partial^{2} \varphi}{\partial z^{2}}=\Delta^{\prime} \varphi=0
$$

By the monotone convergence theorem,

$$
\lim _{R \rightarrow \infty} \int_{\Sigma}-\left(|A|^{2} \varphi^{2}+\kappa \varphi^{2}\right) e^{-\frac{\kappa z^{2}}{2}}=\int_{\Sigma}-\left(|A|^{2}+\kappa\right) e^{-\frac{\kappa z^{2}}{2}}
$$

On the other hand,

$$
\lim _{R \rightarrow \infty} \int_{\Sigma \cap\{R \leq|z| \leq 2 R\}}\left(\frac{2|z|}{R}+\frac{z^{2}}{R^{2}}\right) e^{-\frac{\kappa z^{2}}{2}}=0,
$$

then $F^{\prime \prime}<0$ if we choose $R$ large enough, thus $\Sigma$ is $F$-unstable.

Case 2: $\langle\partial z, \nu\rangle$ changes sign. We denote by $\mu_{1}$ the first eigenvalue of the operator $L$.

Claim. $\mu_{1}<-\kappa$.

Observe that

$$
L\langle\partial z, \nu\rangle=\kappa\langle\partial z, \nu\rangle
$$

and that $\langle\partial z, \nu\rangle$ does not vanish identically. Then by the Lemma 9.25 in [6], we only need to show $\langle\partial z, \nu\rangle$ is in the weighted $W^{1,2}$ space. Since 1 and $|A|$ are in the weighted $L^{2}$ space, and

$$
|\langle\partial z, \nu\rangle| \leq 1,|\nabla\langle\partial z, \nu\rangle| \leq|A|,
$$

then $\langle\partial z, \nu\rangle$ is in the weighted $W^{1,2}$ space.

For a fixed point $p \in \Sigma$, let $B_{r}(p)$ denote the intrinsic ball of radius $r$ and centered at $p$. Since $\mu_{1}<-\kappa$, then we can choose some $r$ large enough, denoted by 
$\bar{r}$, such that the lowest eigenvalue $\mu_{1}\left(B_{\bar{r}}\right)<-\kappa$. Let $u_{\bar{r}}$ be the nonzero solution to the Dirichlet equation:

$$
L u_{\bar{r}}=-\mu_{1}\left(B_{\bar{r}}\right) u_{\bar{r}}
$$

Now let $\varphi=u_{\bar{r}}$; then

$$
\begin{aligned}
F^{\prime \prime} & =\int_{\Sigma}\left(-\varphi L \varphi+2 \kappa h \varphi\langle\partial z, \nu\rangle-\kappa h^{2}\langle\partial z, \nu\rangle^{2}\right) e^{-\frac{\kappa z^{2}}{2}} \\
& =\int_{\Sigma}\left(\mu_{1}\left(B_{\bar{r}}\right) u_{\bar{r}}^{2}+2 \kappa h u_{\bar{r}}\langle\partial z, \nu\rangle-\kappa h^{2}\langle\partial z, \nu\rangle^{2}\right) e^{-\frac{\kappa z^{2}}{2}} \\
& =\int_{\Sigma}\left[\left(\mu_{1}\left(B_{\bar{r}}\right)+\kappa\right) u_{\bar{r}}^{2}-\kappa\left(u_{\bar{r}}-h\langle\partial z, \nu\rangle\right)^{2}\right] e^{-\frac{\kappa z^{2}}{2}} \\
& \leq \int_{\Sigma}\left(\mu_{1}\left(B_{\bar{r}}\right)+\kappa\right) u_{\bar{r}}^{2} e^{-\frac{\kappa z^{2}}{2}} \\
& <0 .
\end{aligned}
$$

\section{REFERENCES}

[1] Xu Cheng, Tito Mejia, and Detang Zhou, Stability and compactness for complete, $f$-minimal surfaces, arXiv:1210.8076[math.DG](2012).

[2] Xu Cheng, Tito Mejia, and Detang Zhou, Eigenvalues estimate and compactness for $f$ minimal surfaces, arXiv:1210.8448[math.DG](2012).

[3] Xu Cheng, Tito Mejia, and Detang Zhou, Simon's type equation for f-minimal hypersurfaces and applications, arXiv:1305.2379[math.DG](2013).

[4] Xu Cheng and Detang Zhou, Volume estimate about shrinkers, Proc. Amer. Math. Soc. 141 (2013), no. 2, 687-696, DOI 10.1090/S0002-9939-2012-11922-7. MR2996973

[5] Tobias Holck Colding and William P. Minicozzi II, A course in minimal surfaces, Graduate Studies in Mathematics, vol. 121, American Mathematical Society, Providence, RI, 2011. MR2780140

[6] Tobias H. Colding and William P. Minicozzi II, Generic mean curvature flow I: generic singularities, Ann. of Math. (2) 175 (2012), no. 2, 755-833, DOI 10.4007/annals.2012.175.2.7. MR2993752

[7] Gerhard Huisken, Asymptotic behavior for singularities of the mean curvature flow, J. Differential Geom. 31 (1990), no. 1, 285-299. MR1030675 (90m:53016)

[8] Gerhard Huisken and Alexander Polden, Geometric evolution equations for hypersurfaces, Calculus of variations and geometric evolution problems (Cetraro, 1996), Lecture Notes in Math., vol. 1713, Springer, Berlin, 1999, pp. 45-84, DOI 10.1007/BFb0092669. MR 1731639 (2000j:53090)

[9] Caleb Hussey, Classification and analysis of low index Mean Curvature Flow self-shrinkers, ProQuest LLC, Ann Arbor, MI, 2012. Thesis (Ph.D.)-The Johns Hopkins University. MR3103611

[10] Debora Impera and Michele Rimoldi, Stability properties and topology at infinity of f-minimal hypersurfaces, arXiv:1302,6160v1[math.DG](2013).

[11] Haizhong Li and Yong Wei, f-Minimal Surface and Manifold with Positive m-Bakry-Émery Ricci Curvature, J. Geom. Anal. 25 (2015), no. 1, 421-435, DOI 10.1007/s12220-013-9434-5. MR.3299288

[12] John Lott, Mean curvature flow in a Ricci flow background, Comm. Math. Phys. 313 (2012), no. 2, 517-533, DOI 10.1007/s00220-012-1503-2. MR2942959

[13] Peter Petersen, Riemannian geometry, 2nd ed., Graduate Texts in Mathematics, vol. 171, Springer, New York, 2006. MR2243772(2007a:53001) 
[14] Yongbing Zhang, $\mathcal{F}$-stability of self-similar solutions to harmonic map heat flow, Calc. Var. Partial Differential Equations 45 (2012), no. 3-4, 347-366, DOI 10.1007/s00526-011-0461-4. MR.2984136

Department of Mathematics, Zhejiang University, Hangzhou 310027, People's RepubLIC OF CHINA

E-mail address: weimins@zju.edu.cn

Department of Mathematics, Zhejiang University, Hangzhou 310027, People's RepubLIC OF CHINA

E-mail address: robin1055@126.com 